\title{
Cinceles en la Edad del Bronce italiana: aspectos tecnológicos y tipológicos
}

\author{
Chisels in the italian Bronze Age: technological and typological aspects
}

\author{
Claudia Speciale $(*)$ \\ Alessandro Zanini $(* *)$
}

\section{RESUMEN}

Los cinceles de bronce son herramientas que se utilizan en percusión apoyada (à percussion posée), por consiguiente, necesitan una longitud mínima y algunas partes funcionales (empuñadura, cuerpo, hoja); podrían tener un mango de material perecedero. Se utilizan con un martillo en el labrado de la madera, del bronce y de otros materiales. Los cinceles se pueden clasificar de acuerdo con los siguientes elementos funcionales: el primero es la forma (cincel de varilla o de cubo); el segundo es la sección del cuerpo; el tercero es la sección de la empuñadura. La cronología y la distribución de cada tipo se identificaron para obtener una visión general de esta clase de instrumentos durante la Edad del Bronce en Italia.

\begin{abstract}
Bronze chisels are tools used "à percussion posée"; this means they need a minimum length and some functional parts (tang, body, blade); they could have a handle of perishable material and they were used with a hammer to work wood, bronze and other materials. Chisels can be classified following their functional parts; the first element is the shape (rod or socketed chisel); the second one is body section; the third one is tang section. Chronology and distribution of every type were identified to obtain a general view of this class of tools during the Italian Bronze Age.
\end{abstract}

Palabras clave: Italia; Edad del Bronce; Herramientas; Cinceles; Tecnología del bronce.

(*) Università del Salento, Scuola di Specializzazione in Beni Archeologici. Via D. Birago 46. Lecce. Italia. Correo electrónico: claudiaspeciale@gmail.com

(**) Università di Palermo, sede di Agrigento, Insegnamento di Preistoria e Protostoria. Via U. La Malfa 1. Agrigento. Italia. Correo electrónico: zanini@dada.it

Recibido: 16-II-2010; aceptado: 14-IV-2010.
Key words: Italy; Bronze Age; Tools; Chisels; Bronze Technology.

\section{INTRODUCCIÓN}

El análisis funcional ocupa un lugar fundamental en la realización de una tipología de instrumentos de trabajo. El estudio ergonómico del objeto, de hecho, permite identificar sus características esenciales y a la vez ayuda a distinguirlo de otras categorías de utensilios con usos afines. Los análisis sobre su composición, asimismo, ayudan a obtener otras respuestas sobre la funcionalidad del instrumento. La realización de la tipología en sí es, pues, una fase fundamental, pero es necesario que la precedan y acompañen una serie de estudios que permitan definir con mayor precisión la entidad de cada clase de utensilios.

En este caso se han considerado los cinceles de la Península Italiana durante la Edad del Bronce para servir de base a la clasificación crono-tipológica.

\section{DEFINICIÓN DE CINCEL}

\subsection{Partes estructurales}

Cabe, por lo tanto, plantearse una cuestión básica: ¿cómo podemos definir un cincel? La escuela antropológica francesa ha sugerido diferentes respuestas; la más exhaustiva sigue siendo la de Leroi-Gourhan (1971: 182-183) (1): une lame à

(1) También se podría citar a Jean Deshayes (1960: 85): outils à tranchant transversal dont les côtés sont à peu près parallèles; la lame peut être plate et étroite, ou épaisse, de section 
tranchant transversal (forme) agissant en percussion posée oblique trasversale, avec percuteur (geste) sur le bois (matière).

Desde un punto de vista estructural, el cincel posee las siguientes partes: una hoja, un cuerpo y una empuñadura (Fig. 1: 1-4); ésta generalmente lleva un mango. Para utilizar un cincel se utiliza un percutor, una marra o un mazo. Dicho instrumento puede tener varias dimensiones, según el tipo de trabajo a efectuar, y ser de varios materiales, generalmente madera o metal (2). El cincel se utiliza en el labrado de la madera, de la piedra, del metal, del cuero, del hueso y de materias duras animales.

Es necesario que la longitud total del cincel, incluyendo el mango, sea suficiente para empuñarlo: considerando el tamaño medio de la mano de un adulto, el instrumento no debería medir menos de 10-12 cm de largo. Como se deduce del gráfico de la figura 2, no existe una neta diferenciación entre cinceles pequeños o grandes; la mayoría de ellos tiene una longitud de 10 a $18 \mathrm{~cm}$, pero los más pequeños miden unos $4-5 \mathrm{~cm}$ y los más largos más de $30 \mathrm{~cm}$. En la mayoría de los casos se conoce sólo la armadura en bronce del cincel, por consiguiente, únicamente se puede estimar su longitud global.

En caso de presencia de un mango, la empuñadura debe tener longitud suficiente para su inserción y para que sostenga el empuje del percutor, incluso en trabajos de pequeña envergadura (Fig. 1: 5). En otras palabras: para que una armadura pueda incorporar un mango de modo estable y el artesano pueda empuñarla cómodamente, la parte de bronce del cincel debería tener por lo menos $5 \mathrm{~cm}$ de largo en total y su mango debería medir al menos $8-10 \mathrm{~cm}$. Una armadura menor que dicha longitud aproximada, pues, no se puede considerar un cincel, sino más bien un instrumento análogo para la realización de labrados sin percusión, como por ejemplo la lezna. Incluso en el

ronde ou plus ou moins carrée; asimismo, en la obra de Nicolardot y Gauchet (1975: 117): outils formés d'une simple lame allongée à tranchant rectiligne perpendiculaire à l'axe longitudinal [...]. Ces outils devaient servir au travail du bois, du métal ou d'autres matières organiques ou minérales (pierre, corne, os et cuir).

(2) Uno de los estudios más exhaustivos sobre cinceles de cobre y de bronce desde el punto de vista experimental se ha llevado a cabo sobre ejemplares egipcios; la morfología y las técnicas de uso eran muy similares a las de los cinceles de la Península Italiana, por ejemplo, los cinceles con empuñadura de material deteriorable, utilizados con un mazo de madera (Stocks 2003). caso de los cinceles de cubo es necesario que la armadura tenga una cierta longitud; son los únicos instrumentos que, claramente, necesitan un mango y efectivamente todos los cinceles de cubo superan los $10 \mathrm{~cm}(3)$.

El mango de los cinceles con empuñadura podía ser de materia dura o madera, fijándose a la parte de bronce por encastre, recurriendo a sustancias para encolar o a pequeños topes situados entre la empuñadura y el mango (4) (Fig. 1: 6). Tampoco se puede excluir que constara sencillamente de una piel o un tejido envueltos alrededor del cincel (Deshayes 1960: 27) (5). En caso de uso de la empuñadura como superficie directa de impacto, el objeto debería presentar señales de desgaste. Éstas, en general, consisten en una serie de pequeñas muescas en los bordes, debidas a los repetidos golpes del percutor (6).

\subsection{Otras herramientas similares}

Un instrumento a menudo clasificado como cincel es el bedano o escoplo (Fig. 1: 7); es también un utensilio de percusión pero con el filo en punta y/o perpendicular al plano de las dos caras del instrumento. Su acción consiste en crear un surco y extraer más fácilmente el material, gracias a la distribución de la fuerza ejercitada, que recae preferentemente en la punta. Lo utiliza principalmente el carpintero (Deshayes 1960: 105), pero también el picapedrero (Rockwell 1989: 41-42, 57, 5.7); más difícil es pensar en su utilización en un taller de toréutica (7).

(3) Los ejemplares más cortos son los de Módica y Niscemi, pero no es segura su función de cinceles (Albanese Procelli 1994: 90).

(4) En el caso de los utensilios de Sant'Anastasia, que es también un taller metalúrgico dentro de la aldea nurágica de la Edad del Hierro, el mango está fijado con pequeñas cuñas de hueso (Ugas y Usai 1987).

(5) En el volumen de los Prähistorische Bronzefunde sobre las hachas de Polonia, el autor se plantea la posibilidad de que fueran como los de los cinceles modernos; seguramente tamaño, peso y función del cincel debían incidir sobre su morfología (Gedl 2004: 73).

(6) En la mayoría de los casos esto se verifica con los cinceles de talón; el ejemplar de Albucciu mide sólo $8 \mathrm{~cm}$ pero tiene un pequeño talón con aparentes señas de desgaste; el cincel de Lavis (Trento) mide sólo $10 \mathrm{~cm}$ pero parece que presenta señales de desgaste provocadas por un instrumento de cabeza redonda (Marzatico 1997: 76); o incluso el fragmento de mango de un utensilio, quizás un cincel, procedente de Castellaro dell'Uscio (Genova) (Maggi 1990: 233-234).

(7) Si bien F. Marzatico (1994: 41-42), por ejemplo, asocia el ejemplar de Segonzano a la elaboración del metal. 

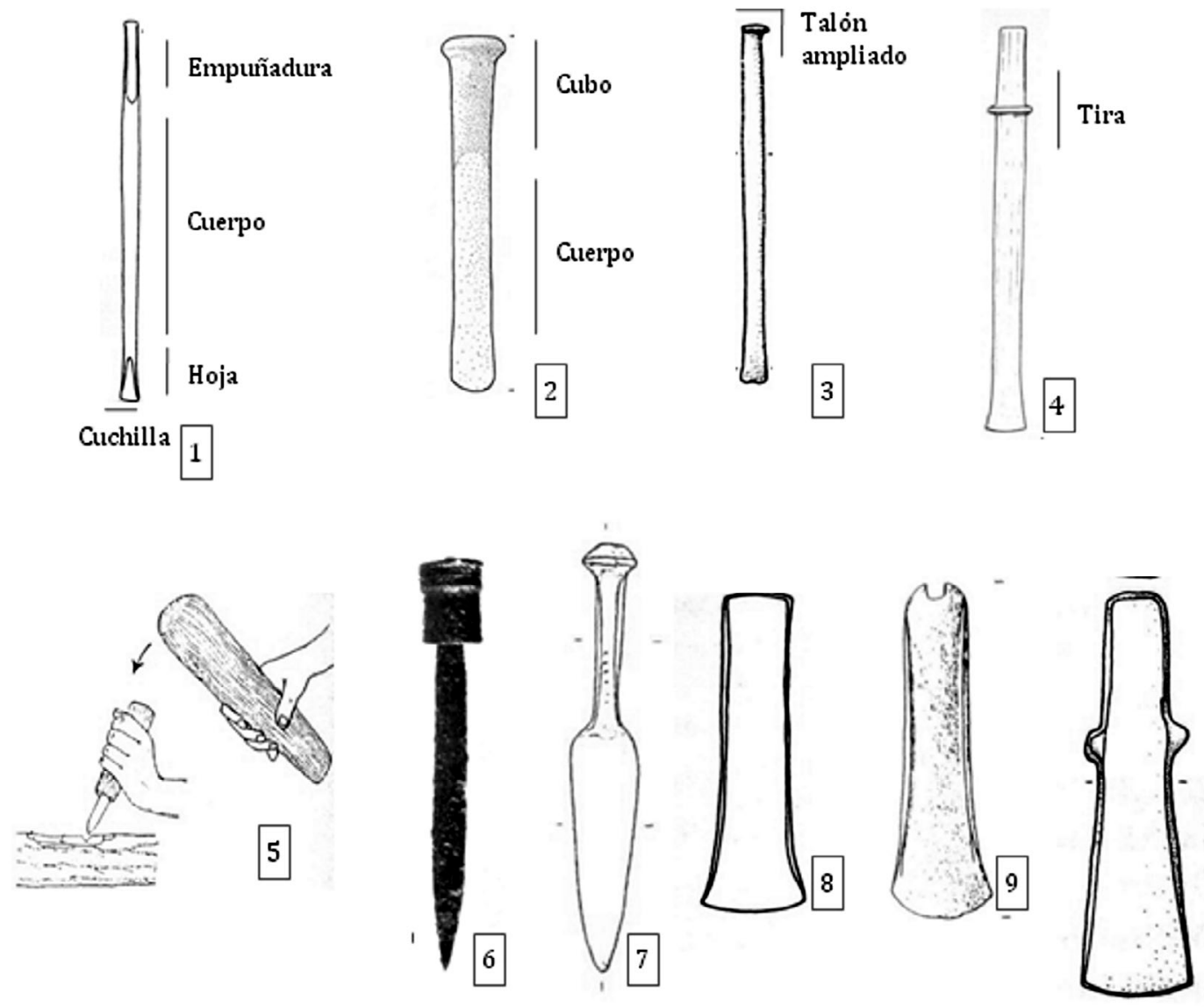

10
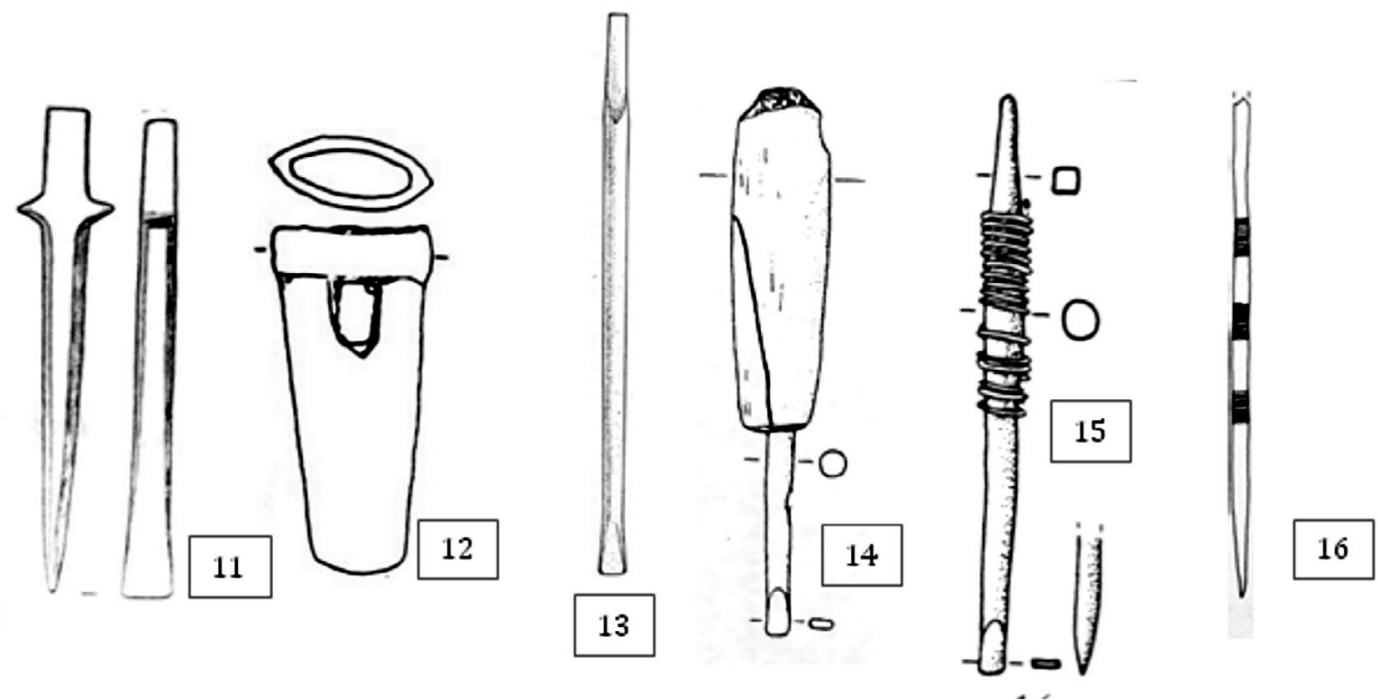

Fig. 1. 1-4. Términos usados en la descripción de las piezas; 5. Uso del cincel (Provenzano 1996-97: Fig. 5: 8); 6. Cincel Sant'Anastasia di Sardara (Ugas y Usai 1987: Tab. IXa); 7. Escoplo Pariana (Cateni 1984: Fig. 9.9); 8. Hacha plana (Lo Schiavo 2004: Fig. 2.1); 9. Baragalla (Bernabò Brea et al. 1997: 307, 1); 10. Hacha de apéndices laterales Decimopetru (Lo Schiavo, D’Oriano 1990: Fig. 3.2); 11. Manduria (Quagliati 1903: Tab. 8, Fig. 7); 12. Hacha de cubo perforado Modica (Giardino 1995: Fig. 10B: 4); Cinceles; 13. Gualdo Tadino (Peroni 1963: Fig. I: 6.30); 14-15. Montale (Bernabò Brea et al. 1997: Fig. 331: 16-17); 16. Castellaro del Vhò (Frontini 2001: Fig. 69: 7). 
Longitud cm

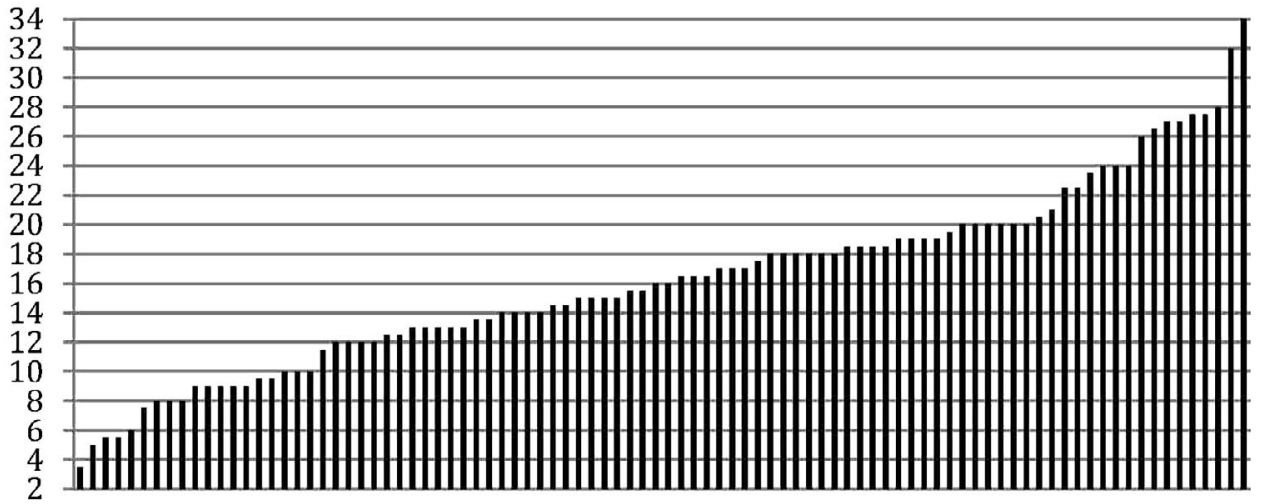

Fig. 2. Gráfico con la longitud de todos los cinceles de la Península Italiana fechados entre la Edad del Bronce Medio y la Primera Edad del Hierro, ordenados de menor a mayor tamaño.

No es de extrañar que, en la mayoría de los volúmenes de los Prähistorische Bronzefunde dedicados a las hachas, una sección se reserve a los cinceles. El hacha requiere un tipo diferente de trabajo, puesto que se trata de un instrumento de percusión directa o lanzada, y no indirecta con percutor como el cincel (8). Por consiguiente los dos instrumentos se diferencian, pero desafortunadamente esto ocurre sobre todo a nivel del mango (9). La exigencia de hallar índices de referencia para distinguir al cincel de las hachas ya se conoce en Italia, en particular por su semejanza con las hachas planas (Lo Schiavo 2004: 238, n. ${ }^{\circ}$ 3; Albanese Procelli 2006: 184). Dicho tipo de hacha, junto con la de bordes levemente realzados, morfológicamente está muy cercano al cincel (10) (Fig. 1: 8-9). Según Albanese Procelli (1996: 120) las hachas planas se podían usar como cinceles para el labrado de la madera, mientras que las hachas de enmangue tubular se usaban para la tala y los grandes trabajos de carpintería. En la introducción del volumen dedicado a las hachas de Francia meridional, se trata el

(8) Por este motivo es necesario excluir que los instrumentos con empuñadura de ángulo recto procedentes del déposito de Niscemi fueran cinceles: aun teniendo una hoja bastante estrecha, se debían de utilizar como instrumentos de percusión lanzada (Albanese Procelli 1994: Fig. 17, NA4; Giardino 1995: Fig. 9: 4-5).

(9) Es muy interesante la observación de Deshayes (1963: 168) que mediante paralelos etnológicos demuestra que las cuñas y las hachas de los carpinteros iraníes serían idénticas si no fuera por los mangos de material perecedero.

(10) Véanse, por ejemplo, los instrumentos definidos como cinceles del depósito de Funtana Janna de Bonnanaro (Lo Schiavo 2000: 169-170). problema de la distinción entre hacha y cincel, identificando algunas características al respecto: el ancho máximo del cincel generalmente no se pone en la hoja, asimismo, es más fino que el hacha y con dimensiones absolutas inferiores. Los autores, de todas formas, admiten la existencia de formas intermedias de interpretación no definitiva, así como de hachas utilizadas secundariamente como cinceles (Chardenoux y Courtois 1979: 7-8). Por otra parte, el mismo Peroni (1994: 80) piensa que los cinceles proceden justamente de las hachas. Son sobre todo los ejemplares de morfología y dimensiones intermedias entre las dos clases los que plantean más interrogantes desde el punto de vista tipológico (11). Un tipo muy particular de hacha -la de apéndices- probablemente utiliza el mismo sistema de fijación que los cinceles con empuñadura en forma de $\mathrm{T}$, aunque la distribución es totalmente diferente (12) (Figs. 1: 10-11, 3: 14). Los instrumentos con cubo perforado, característicos de algunos depósitos sicilianos, no se han interpretado unívocamente (Fig. 1: 12): de hecho es posible identificarlos como hachas y cinceles, aunque no se puede descartar la hipótesis de que se utilizaran como rejas de arado (13).

(11) Por ejemplo, la pieza de Baragalla, definida como hacha-cincel (Bernabò Brea et al. 1997: 307; De Marinis 1976: 232-233)

(12) Las hachas con apéndices laterales son una producción característica de Sicilia, Cerdeña, España, con una excepción en el Tirreno central como Monte Rovello (Giardino 1995: 200-205); los cinceles con empuñadura en forma de T, en cambio, son conocidos en Etruria y Pulla.

(13) Véase nota 3. 


\subsection{Nota funcional}

Durante el proceso de identificación de los instrumentos de trabajo, cabe recordar que un utensilio de forma similar o idéntica puede servir para funciones totalmente diferentes. Es suficiente confrontar un destornillador moderno plano con algunos de los cinceles de tipo Surbo para darse cuenta de que una misma forma se presta en teoría a diferentes interpretaciones funcionales -en este caso cincelar y atornillar (14) (Fig. 1: 13)-. Si se observa el primero de los cinceles de Montale, por ejemplo, se notará un fino alambre de bronce enroscado alrededor del cuerpo (Fig. 1: 15). No es posible decir con seguridad si servía para la fijación del mango o para que el instrumento hiciera un movimiento diferente de la cinceladura, aunque la presencia de otro cincel con armadura idéntica y mango de hueso más bien permite pensar en un uso diferente de los dos objetos (Fig. 1: 14). El fino cincel de tipo Cevola de Castellaro del Vhò (Cremona) presenta en el cuerpo tres haces de ranuras, pero es imposible decir si servían, por ejemplo, para que el instrumento efectuara un movimiento de rotación (15) (Fig. 1: 16).

\section{FUNDAMENTOS DE LA TIPOLOGÍA}

\subsection{Criterios de clasificación}

La tipología realizada se basa ante todo en la distinción entre dos clases de cinceles: de barra y de cubo. Los primeros se subdividen luego en una serie de formas funcionales a partir de la sección del cuerpo; los cinceles de sección circular, cuadrangular, octogonal o rectangular plana se subdividen a su vez por el tipo de mango en diferentes grupos, definidos aquí como "tipos" y denominados a partir del ejemplar más representativo. Los cinceles de cubo se subdividen según la relación entre mango y cuerpo del cincel (distinto/indistinto) y por consiguiente en base a la presencia/ausencia del propio mango. Se han identifica-

(14) Puesto que el destornillador y los tornillos se inventaron sólo a finales de la Edad Media (Rybczynski 2000), seguramente aún no era necesario que un utensilio tuviera la función de atornillar.

(15) Pero también se puede plantear otra hipótesis: que el utensilio se haya obtenido de la reelaboración de un punzón; por ejemplo, la pieza 961 de la clasificación del volumen de la Prähistorische Bronzefunde (Frontini 2001: Fig. 69.7), de Castellaro del Vhò, presenta el mismo tipo de decoraciones. do así 112 cinceles reagrupados en 14 tipos, que se ilustran en la figura 3 y se resumen en las tablas 1 y 2; se han identificado asimismo algunos unica ( 5 en total), o bien ejemplares cuya morfología no se corresponde con los citados tipos.

\subsection{Cuadro cronológico y geográfico de los tipos}

Se deduce, pues, un cuadro bastante claro para la Península Italiana en lo relativo a la cronología (Fig. 4) y la distribución geográfica (Fig. 5).

El proceso de formación de la herramienta "cincel" arrancó en Europa Centro-oriental desde el comienzo de la Edad del Bronce (véase por ejemplo Laux 2000: 67), pero en Italia sólo aparecen más tarde. Como se explica en el apartado 2.2, parece que el cincel es una forma que se originó durante la Edad del Bronce Antiguo a partir de la clase de las hachas, con las que también asemeja tener en lo sucesivo muchas afinidades tipológicas. Entre la segunda fase de la Edad del Bronce Medio (1600-1500 BC) y el Bronce Reciente (1350-1200 BC) se desarrollan los primeros tipos, sobre todo en Emilia Romaña, Lombardía y Véneto (Cevola, Gorzano, Ognissanti) (Fig. 3: 1-2, 4); más incierta es la atribución a esta fase del ejemplar de Capo Graziano, en las Islas Eolias (Bernabò Brea y Cavalier 1991: tav. CIV, 6). La estructura de estos cinceles es bastante simple, con cuerpo de sección circular o cuadrangular indistinto de la empuñadura. Sólo en el Bronce Reciente se desarrolla el tipo más elaborado Chilivani, de grandes dimensiones y sección octogonal. Al final de este período, que corresponde a la desaparición de la cultura de Terramare, es posible hallar cinceles en muchas regiones: Trentino, Friul, Cerdeña, Umbría, Pulla (Surbo, Cortine, Gruxi 'e Crobu) (Fig. 3: 3, 5, 7). La forma es más elaborada y las dimensiones de muchos de ellos son de unos $25 \mathrm{~cm}$. En la fase 1 del Bronce Final (1200-1100 BC) la producción de cinceles decrece, exceptuando en Cerdeña, y hay pocos ejemplares en Sicilia y Friul (Chilivani, Noto, Lestizza) (Fig. 3: 8, 10-11); al final de esta fase aparece el primer cincel de cubo italiano. En el Bronce Final 2 (1100-1020/1000 BC), la Etruria septentrional e interna son las zonas principales de producción: entre los modelos más característicos, el tipo Bagnolo presenta un tope entre cuerpo y empuñadura y un área de difusión 

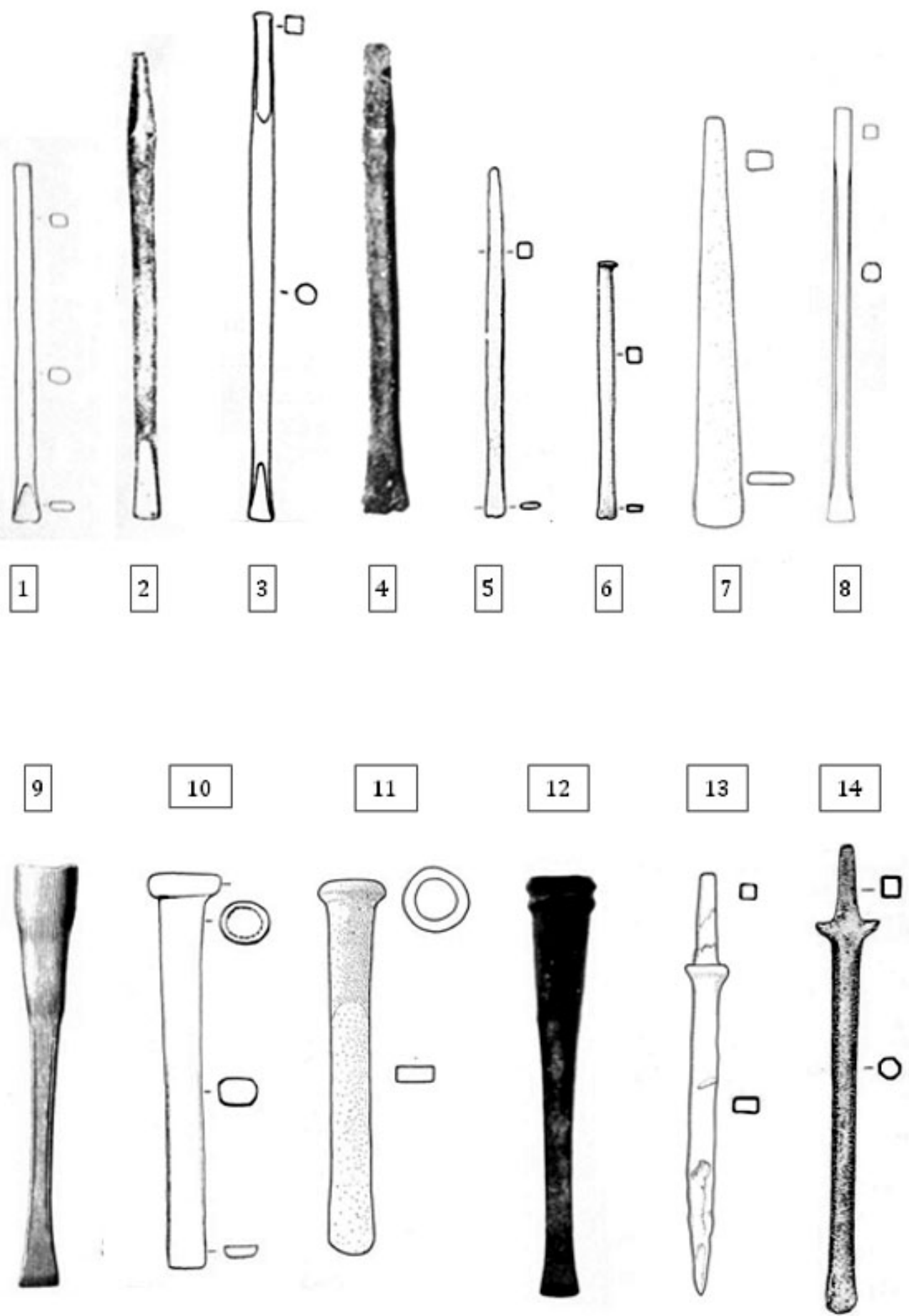

Fig. 3. Tipos epónimos de cinceles de la Península Italiana fechados entre la Edad del Bronce Medio y la Primera Edad del Hierro: 1. Cevola (Mutti 1994: Fig. 131: 6); 2. Gorzano (Säflund 1939: Tab. 4: 11); 3. Surbo (Bietti Sestieri 1973: Fig. 1: 3); 4. Ognissanti (Barocelli 1971: Fig. 24: 25); 5. Cortine di Fabriano (Lollini 1979: Fig. 1: 4); 6. Redù (Carancini 1997: Fig. 331: 15); 7. Gruxi ‘e Crobu (Lo Schiavo et al. 2004: Fig. 4, 10); 8. Chilivani (Lo Schiavo 1989: Tab. 4: 10); 9. Corinaldo (Montelius 1895-1910: Tab. 125: 10); 10. Noto (Albanese Procelli 1993: Tab. 8, NA5); 11. Lestizza (Borgna 2000: Fig. 14: 2); 12. Francavilla Marittima (Zancani Montuoro 1977: Fig. 21); 13. Bagnolo (Balducci, Lo Schiavo, Zanini en prensa); 14. "Fra Gabbro e Colognole" (Zanini 1997: Fig. 133: 6).

T. P., 67, N. ${ }^{\circ}$ 2, julio-diciembre 2010, pp. 419-430, ISSN: 0082-5638 doi: $10.3989 /$ tp.2010.10048 


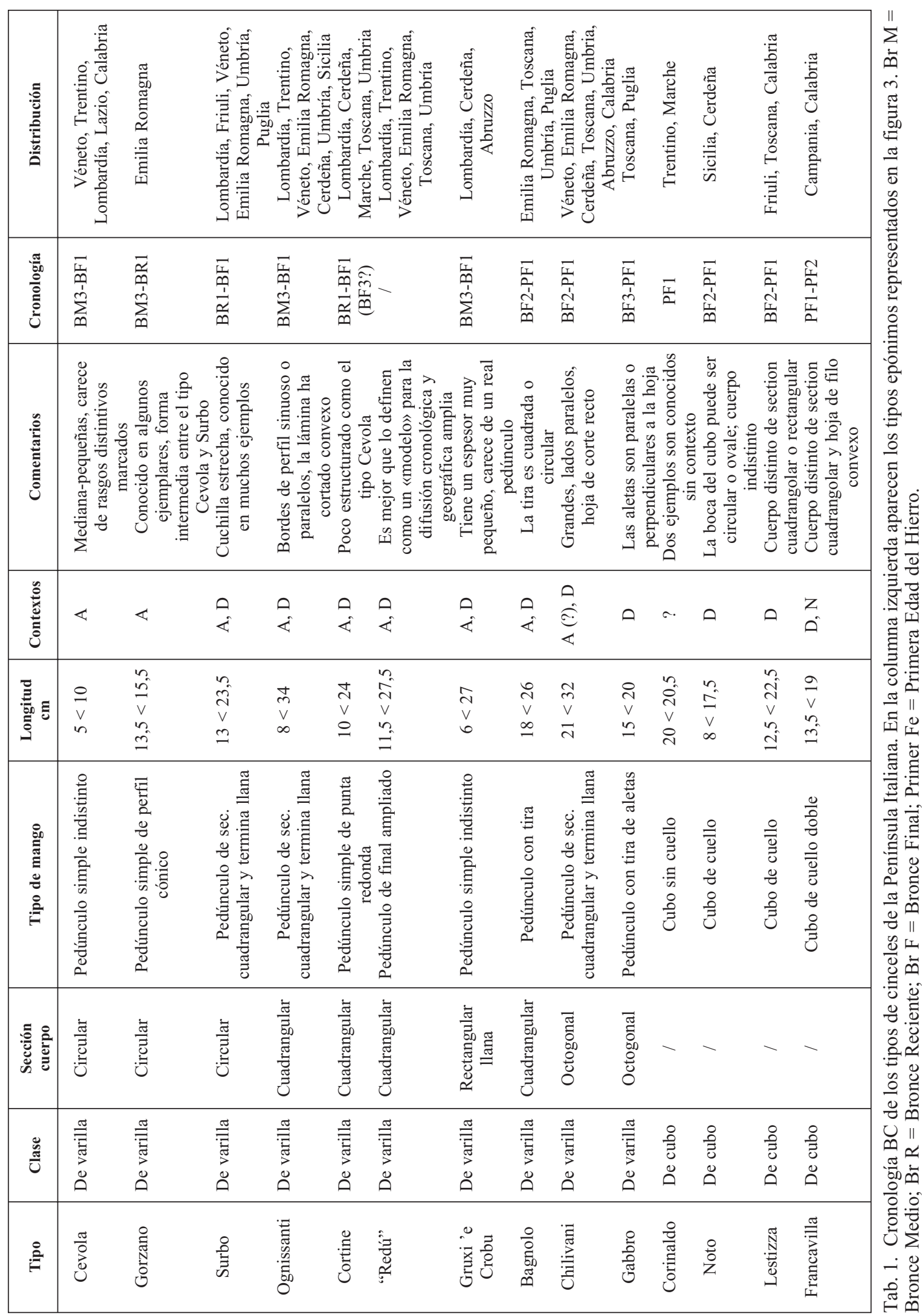

T. P., 67, N. ${ }^{\circ} 2$, julio-diciembre 2010, pp. 419-430, ISSN: 0082-5638 doi: $10.3989 /$ tp.2010.10048 


\begin{tabular}{|c|c|c|c|}
\hline Tipo & Otros especímenes & Distribución & Nombre \\
\hline Cevola (Parma) (1) & $\begin{array}{l}\text { Castellaro del Vhò (Cremona); Muraiola } \\
\text { (Verona); Madrarosa (Vicenza); Broglio di } \\
\text { Trebisacce (Cosenza); Sorgenti della Nova } \\
\text { (Viterbo); Romagnano (Trento) }\end{array}$ & $\begin{array}{c}\text { Veneto, Trentino, } \\
\text { Lombardia, Lazio, Calabria }\end{array}$ & 7 \\
\hline Gorzano (Modena) (2) & $\begin{array}{c}\text { Montale (Modena); Castione dei Marchesi } \\
\text { (Reggio Emilia) }\end{array}$ & Emilia Romagna & 4 \\
\hline Surbo (Lecce) (3) & $\begin{array}{l}\text { Gualdo Tadino (Perugia); San Polo d'Enza } \\
\text { (Reggio Emilia); Campeggine (Reggio Emilia); } \\
\text { Ognissanti (Cremona); Castions di Strada B } \\
\text { (Udine); Poggio Berni (Rimini); Trebbio Sei Vie } \\
\text { (Bologna); Castellaro Gottololengo (Brescia); } \\
\text { Frattesina (Rovigo); Scoglio del Tonno (Taranto) }\end{array}$ & $\begin{array}{c}\text { Lombardia, Friuli, Veneto, } \\
\text { Emilia Romagna, Umbria, } \\
\text { Puglia }\end{array}$ & 14 \\
\hline $\begin{array}{c}\text { Ognissanti } \\
\text { (Cremona) (4) }\end{array}$ & $\begin{array}{l}\text { Imboccatura del Mincio (Verona); Sa Mandra ‘e } \\
\text { Sa Giua (Sassari); San Polo D’Enza (Reggio } \\
\text { Emilia); Sedda Ottinnnera (Sassari); Chilivani } \\
\text { (Sassari); Sabbionara Veronella (Verona); Lavis } \\
\text { (Trento); Monte Sa Idda (Cagliari); Lipari } \\
\text { (Messina) }\end{array}$ & $\begin{array}{l}\text { Lombardia, Trentino, } \\
\text { Veneto, Emilia Romagna, } \\
\text { Sardegna, Umbria, Sicilia }\end{array}$ & 17 \\
\hline $\begin{array}{l}\text { Cortine di Fabriano } \\
\text { (Ancona) (5) }\end{array}$ & $\begin{array}{c}\text { Serra Orrios (Nuoro); Palmavera (Sassari); } \\
\text { Castellaro Lagusello (Mantova); Cisano (Verona); } \\
\text { Monte Ingino (Perugia); Goluzzo (Siena); Capo } \\
\text { Graziano (Messina); Mendolito (Catania) }\end{array}$ & $\begin{array}{c}\text { Lombardia, Veneto, } \\
\text { Sardegna, Marche, Toscana, } \\
\text { Umbria, Sicilia }\end{array}$ & 9 \\
\hline Redù (Modena) (6) & $\begin{array}{l}\text { Tragno (Trento); Cornocchio (Parma); Isolone del } \\
\text { Mincio (Mantova); Campeggine (Reggio Emilia); } \\
\text { Imboccatura del Mincio (Verona); Cisano } \\
\text { (Verona); Piediluco I (Terni); Campese (Grosseto) }\end{array}$ & $\begin{array}{l}\text { Trentino, Veneto, Emilia } \\
\text { Romagna, Toscana }\end{array}$ & 9 \\
\hline $\begin{array}{c}\text { Gruxi e' Crobu } \\
\text { (Medio Campidano) } \\
\text { (7) }\end{array}$ & $\begin{array}{c}\text { Sedda Ottinnera (Sassari); Santa Caterina } \\
\text { (Cremona); Isolone del Mincio (Mantova); } \\
\text { Albucciu (Olbia); Fucino (L’Aquila) }\end{array}$ & $\begin{array}{l}\text { Lombardia, Sardegna, } \\
\text { Abruzzo }\end{array}$ & 8 \\
\hline Bagnolo (Siena) (13) & $\begin{array}{c}\text { Mottola (Taranto); Piediluco I y II (Terni); } \\
\text { Limone I (Livorno); Trebbo Sei Vie (Bologna); } \\
\text { Casalecchio di Reno (Bologna); Leporano } \\
\text { (Taranto) }\end{array}$ & $\begin{array}{l}\text { Emilia Romagna, Toscana, } \\
\text { Umbria, Puglia }\end{array}$ & 12 \\
\hline Chilivani (Sassari) (8) & $\begin{array}{c}\text { Sedda Ottinnera (Sassari); Poggio Berni (Rimini); } \\
\text { Monte Sa Idda (Cagliari); Piediluco II (Terni); “A } \\
\text { sud di Crotone" (Crotone); Limone I (Livorno); } \\
\text { Colle LE Banche (Livorno); Frattesina (Rovigo); } \\
\text { Monte Lozzo (Padova); Pinerolo (Torino); } \\
\text { Ortucchio (L'Aquila) }\end{array}$ & $\begin{array}{l}\text { Veneto, Emilia Romagna, } \\
\text { Sardegna, Toscana, Umbria, } \\
\text { Abruzzo, Calabria }\end{array}$ & 17 \\
\hline Gabbro (Livorno) (14) & $\begin{array}{c}\text { Pariana (Massa); Manduria I (Taranto); Goluzzo } \\
\text { (Siena) }\end{array}$ & Toscana, Puglia & 4 \\
\hline $\begin{array}{l}\text { Corinaldo } \\
\text { (Ancona) (9) }\end{array}$ & S. Maria Maddalena (Trento) & Trentino, Marche & 2 \\
\hline $\begin{array}{l}\text { Noto Antica } \\
\text { (Siracusa) (10) }\end{array}$ & Niscemi (Caltanissetta); Modica (Ragusa) & Sicilia & 4 \\
\hline Lestizza (Udine) (11) & Limone I (Livorno); “A sud di Crotone” (Crotone) & Friuli, Toscana, Calabria & 4 \\
\hline $\begin{array}{c}\text { Francavilla } \\
(\text { Cosenza) }(12)\end{array}$ & Pontecagnano (Salerno) & Calabria, Campania & 3 \\
\hline
\end{tabular}

Tab. 2. Cuadro con el número y distribución de ejemplares de cada tipo de cincel de la Península Italiana. En la columna izquierda, los nombres y números entre paréntesis se refieren a la figura 3. 


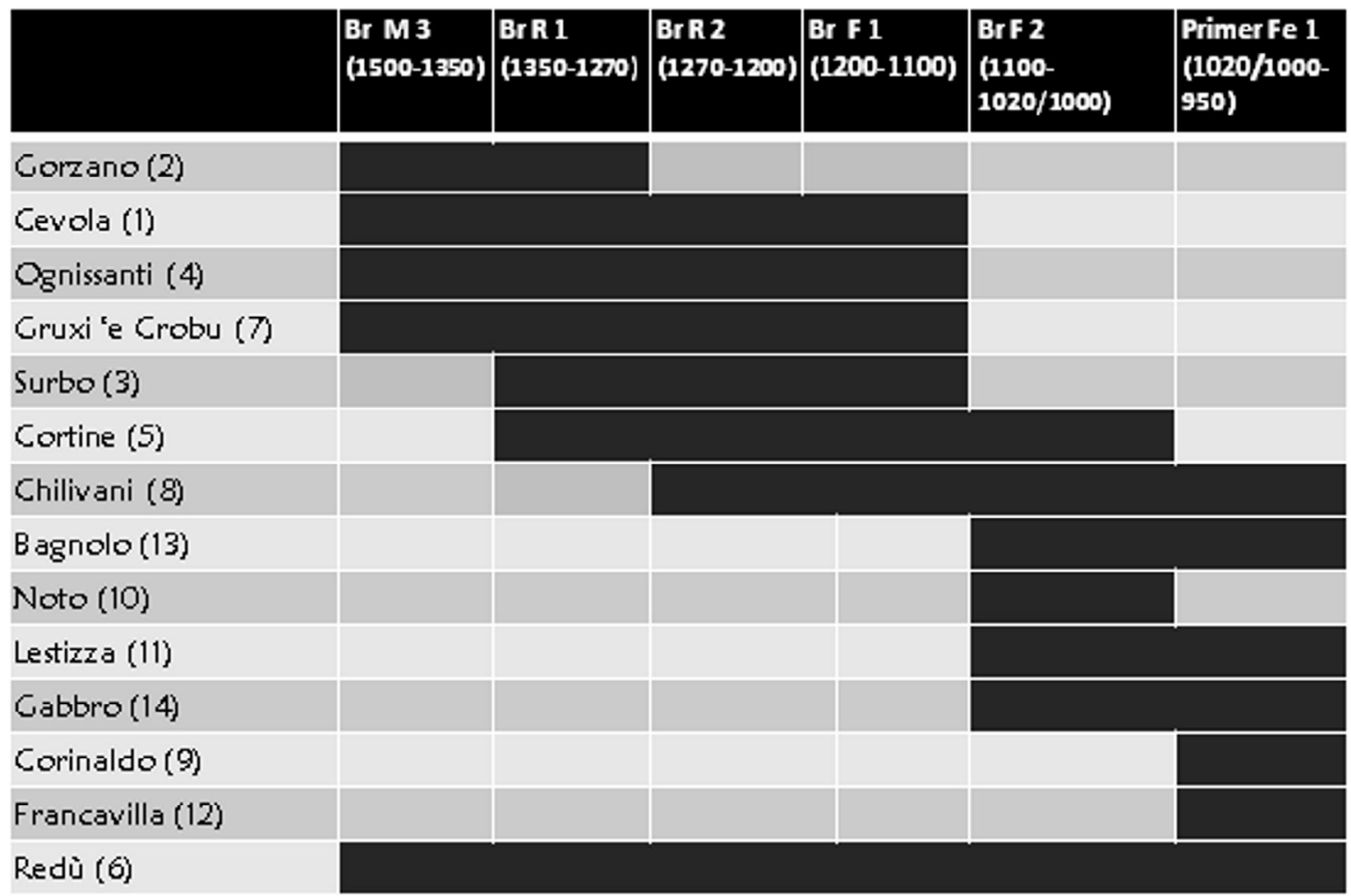

Tab. 3. Cuadro de resumen de cada tipo; los nombres de tipos se refieren a la figura 3; en "Contextos", $\mathrm{A}=$ Asentamiento, $\mathrm{D}=$ Depósito, $\mathrm{N}=$ Necrópolis.

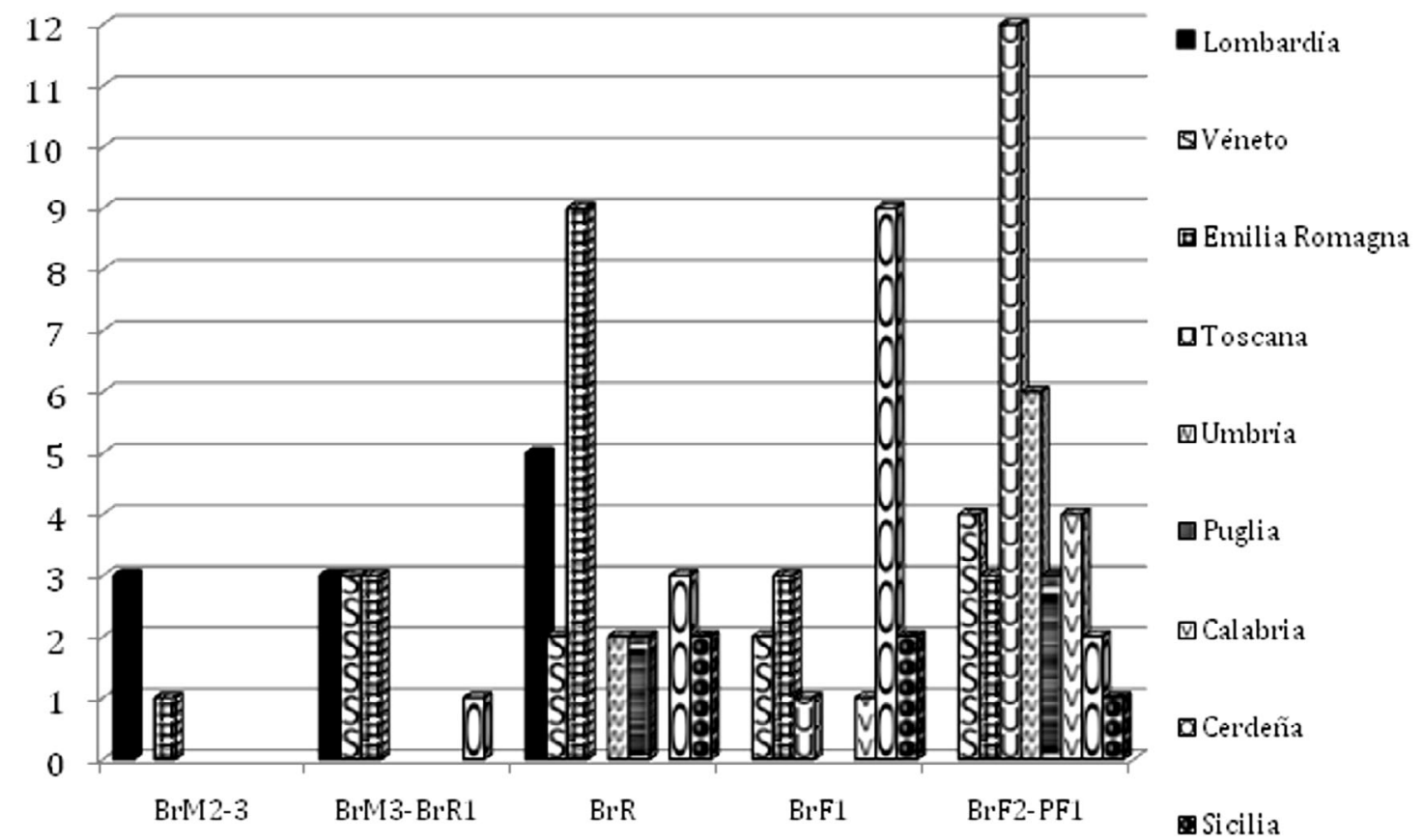

Fig. 4. Cronología BC de los tipos de cinceles de la Península Italiana. En la columna izquierda aparecen los tipos epónimos representados en la figura 3. Br M = Bronce Medio; $\mathrm{Br} \mathrm{R}=$ Bronce Reciente; $\mathrm{Br} \mathrm{F}=\mathrm{Bronce}$ Final; Primer $\mathrm{Fe}=$ Primera Edad del Hierro. 


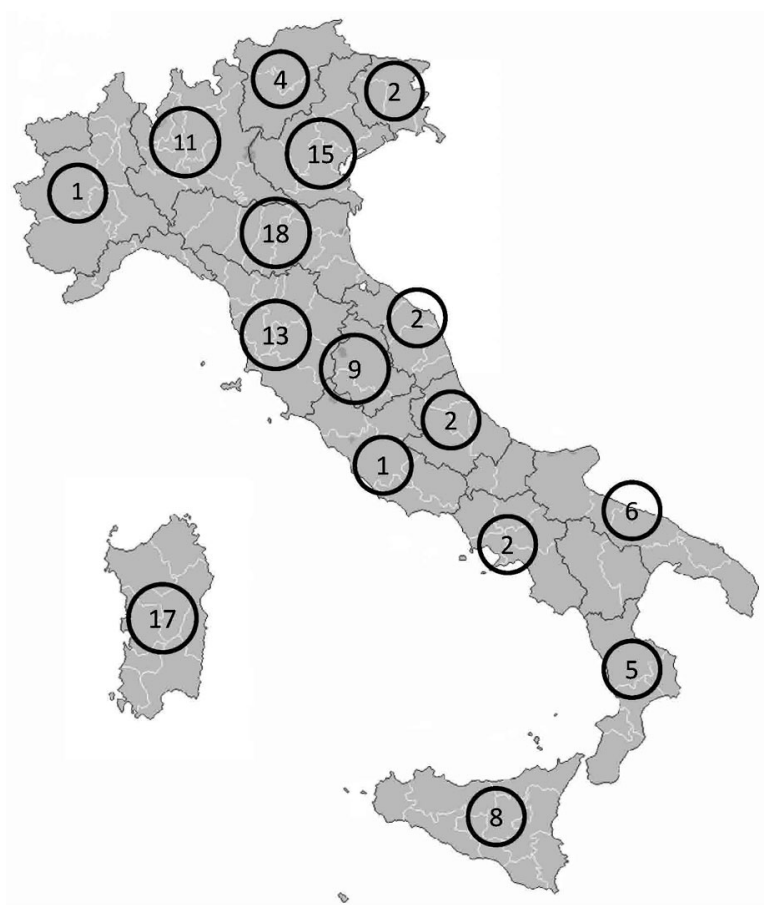

Fig. 5. Distribución del número total de cinceles por regiones italianas (Edad del Bronce Medio a la Primera Edad del Hierro).

que llega hasta Pulla (Fig. 3: 13). Finalmente, a inicios de la Edad del Hierro (1020/1000-950 BC) se desarrollan tipos específicos (Gabbro, con la empuñadura con aletas, y los tipos de cubo Corinaldo y Francavilla) (Fig. 3: 14, 9, 12), reflejo, también para esta clase de manufacturas, de la regionalización de las esferas de acción metalúrgicas. Es importante señalar que el Italia Nord, el noroeste de Italia (Liguria, Piamonte y Valle de Aosta) y las regiones centro-orientales permanecen fuera del fenómeno de difusión de los cinceles, así como, en el lado del Mar Tirreno, Lazio y Campania, este último por lo menos hasta la Edad del Hierro. Obviamente, todo se explica por razones históricas y no sólo por la falta de evidencias arqueológicas, y debe estar conectado con la actividad artesanal de la madera y el metal.

\subsection{Contextos de descubrimiento}

Como se deduce del gráfico de la tabla 1 , los cinceles se encuentran exclusivamente en contextos de asentamiento o en depósitos. Los tipos más antiguos, que se distribuyen entre el valle inferior del Mincio (Lombardía y Véneto) y el distrito de
Módena (Emilia Romagna) (Fig. 5), aparecen sólo en poblados. Desde el Bronce Reciente en toda la península los cinceles comienzan a estar, incluso dentro de depósitos, siempre asociados con otros instrumentos, sobre todo hachas. En el Bronce Final 1, período que, en toda la península, es menos rico en recuperación de metal, Cerdeña en particular destaca por depósitos que contienen muchas herramientas, como Sedda Ottinnera y Chilivani (Lo Schiavo 1989, 1999). Los depósitos son los contextos exclusivos de descubrimiento en el Bronce Final 2; los grandes depósitos que contienen una amplia gama de productos de metal se concentran entre el final de esta fase y la primera Edad del Hierro en Toscana, Umbría y la baja Emilia Romagna. Si nos fijamos en los abundantes depósitos del territorio italiano, vemos que los que incluyen cinceles son pocos (menos de treinta sobre centenares conocidos en la península y las islas en toda la Edad del Bronce y la Primera Edad del Hierro). Se ha hecho hincapié, en lo que respecta a los contextos de descubrimiento, en la ausencia de cinceles y herramientas, hachas incluidas, en las tumbas hasta principios de la Edad de Hierro. Parece que antes las herramientas de trabajo no se consideran un elemento de caracterización de las tumbas masculinas. Esto podría explicarse o por la ausencia de artesanos de la madera, a diferencia de la figura del metalúrgico que parece bien definida socialmente al menos desde la Edad del Bronce tardía, o por la elección de no caracterizar a los difuntos en la tumba por sus actividades productivas 0 , en general, por una falta de identificación de la artesanía como actividades de las elites, como ocurre en algunas tumbas principescas de la Edad del Bronce de Europa centro-septentrional (Kristiansen y Larsson 2005: 57-58).

\section{BIBLIOGRAFÍA}

Albanese Procelli, R.M. 1993: Ripostigli di bronzi della Sicilia nel Museo Archeologico di Siracusa. Accademia di Scienze, Lettere e Arti. Palermo.

Albanese Procelli, R.M. 2004: "La metallurgia del bronzo in Sicilia in età protostorica". En A. Lehoërff (ed.): L'artisanat metallurgique dans les sociétés anciennes en Méditerranée Occidentale. Techniques, lieux et formes de production. École française de Rome. Roma: 195-209.

Aspes, A. 1984 (ed.): Il Veneto nell'antichità. Preistoria e Protostoria. Banca Popolare di Verona. Verona. 
Balducci, C.; Lo Schiavo, F. y Zanini, A. (e.p.): "L'area chiusina fra la fine del mondo terramaricolo e i nuovi assetti medio-tirrenici. Lo scavo di Bagnolo". IX Incontro di studi di Preistoria e Protostoria in Etruria, Valentano/Pitigliano 2008.

Barocelli, P. 1971: Il Castellaro di Gottolengo. Ateneo. Brescia.

Bernabò Brea, L. y Cavalier, M. 1991 (eds.): Meligunis Lipara vol. VI. Filicudi insediamenti dell'età del Bronzo. Accademia di Scienze e Lettere. Palermo.

Bernabò Brea, M.; Cardarelli, A. y Cremaschi, M. 1997 (eds.): Le Terramare. La più antica civiltà padana. Electa. Milano.

Bietti Sestieri, A.M. 1973: "The metal industry of continental Italy, 13th to the 11 th century BC, and its connections with the Aegean". Proceedings of the Prehistoric Society 39: 383-424.

Borgna, E. 2001: "I ripostigli del Friuli. Proposta di seriazione cronologica e di interpretazione funzionale". Rivista di Scienze Preistoriche 51: 289-335.

Carancini, G.L. 1997: "La produzione metallurgica delle terramare nel quadro dell'Italia protostorica". En M. Bernabò Brea, A. Cardarelli, M. Cremaschi (eds.): Le Terramare. La più antica civiltà padana. Electa. Milano: 379-404.

Cateni, G. 1984: "Il ripostiglio di Pariana". En M.C. Marzi Costagli y L.G. Tamagno Perna (eds.): Studi di antichità in onore di Guglielmo Maetzke 1. Bretschneider. Roma: 19-29.

Chardenoux, M.-B. y Courtois J.-C. 1979: Les haches dans la France Méridionale. Prähistorische Bronzefunde 9, 11. C.H. Beck. München.

De Marinis, R. 1976: “Il ripostiglio dell'antica età del Bronzo della Baragalla presso Reggio Emilia”. En Atti della 19a Riunione Scientifica dell'Istituto Italiano di Preistoria e Protostoria (Emilia e Romagna 1975). Firenze: 213-242.

Deshayes, J. 1960: Les outils de bronze, de l'Indus au Danub (IVe au II Millénaire. Librairie Orientaliste Paul Geuthner. Paris.

Deshayes, J. 1963: “Outils de l'Age du Bronze et Economie". En P. Courbin (ed.): Études Archéologiques. Recueil de Travaux. Sevpen. Paris: 167-173.

Frontini, P. 2001 (ed.): Castellaro del Vhò. Campagne di scavo 1996-1999: scavi delle Civiche raccolte Archeologiche di Milano. New Press. Como.

Gedl, M. 2004: Die Beile in Polen IV (Metalläxte, Eisenbeile, Hämmer, Ambosse, Meißel, Pfrieme). Prähistorische Bronzefunde 9, 24. Franz Steiner. Stuttgart.

Giardino, C. 1995: Il Mediterraneo Occidentale fra XIV ed VIII sec. a.C. Cerchie minerarie e metallurgiche. British Archaeological Reports, International Series 612. Archaeopress. Oxford.
Kristiansen, K. y Larsson, T.B. 2005: The rise of Bronze Age Society: travels, transmissions and transformations. Cambridge University Press. Cambridge.

Laux, F. 2000: Die Äxte und Beile in Niedersachsen I (Flach-, Randleisten- und Absaztbeile). Prähistorische Bronzefunde 9, 23. Franz Steiner. Stuttgart.

Leroi-Gourhan, A. 1971: Evolution et techniques I. L'homme et la matière. A. Michel. Paris.

Lollini, D. 1979: "Il Bronzo Finale nelle Marche". Rivista di Scienze Preistoriche 34: 79-214.

Lo Schiavo, F. 1989: "Il ripostiglio di Chilivani, Ozieri (Sassari)". Quaderni della Soprintendenza Archeologica per le province di Cagliari e Oristano 5 (1988): 77-90.

Lo Schiavo, F. y D’Oriano, R. 1990: “La Sardegna sulle rotte dell'Occidente". En La Magna Grecia e il lontano Occidente. Atti del XXIX Convegno di Studi sulla Magna Grecia (Taranto 1989). Napoli: 99-161.

Lo Schiavo, F. 1999: "I lingotti oxhide nel Mediterraneo ed in Europa centrale". En V. La Rosa, D. Palermo y L. Vagnetti (coords.): Epi ponton plazomenoi. Simposio italiano di studi egei (Roma, 1998). Roma: 499-518.

Lo Schiavo, F. 2000: Bronzi e bronzetti del Museo "G.A. Sanna di Sassari”. Imago Media. Piedimonte Matese.

Lo Schiavo, F. 2004: "Produzione, distribuzione e conservazione degli strumenti da lavoro nella Sardegna nuragica". En A. Lehoerff (ed.): L'artisanat métallurgique dans les sociétés anciennes en Méditerranée occidentale. Techniques, lieux et formes de production (Ravello, 2000). Collection de l'École Française de Rome. Roma: 229-261.

Lo Schiavo, F. et al. 2004: "La Sardegna. Articolazioni cronologiche e differenziazioni locali. La metallurgia”. En D. Cocchi Genick (ed.), L'età del Bronzo Recente in Italia. Atti del Congresso Nazionale (Lido di Camaiore, 2000). Viareggio: 357-382.

Maggi, R. 1990 (ed.): Archeologia dell'Appennino Ligure. Gli scavi del Castellaro di Uscio: un insediamento di crinale occupato dal Neolitico alla conquista romana. Istituto internazionale di studi liguri. Bordighera.

Marzatico, F. 1994: "I ritrovamenti arcaici di Cembra nel quadro dell'antico popolamento della valle". Storia di Cembra, cap. 2. Panorama. Trento: 39-59.

Marzatico, F. 1997 (ed.): I materiali preromani della Valle dell'Adige nel Castello di Buonconsiglio I-III. Servizio beni culturali della Provincia autonoma. Trento.

Montelius, O. 1895-1910: La Civilisation primitive en Italie depuis l'introduction des métaux. Imprimerie Royale. Stockholm.

Mutti, A. 1994: Caratteristiche e problemi del popolamento terramaricolo in Emilia Occidentale. University Press. Imola. 
Nicolardot, J.-P. y Gaucher, G. 1975: Typologie des objets de l'Age du Bronze en France V: Outils. Imprimerie Louis-Jean. Paris: 3-4, 117-125.

Peroni, R. (ed.) 1963: "Ripostigli dell'Appennino umbro-marchigiano". Inventaria Archeologica 3. Sansoni. Firenze.

Peroni, R. 1994: Introduzione alla protostoria italiana. Laterza. Roma.

Provenzano, N. 1996-97: "Per una definizione della tecnologia ossea nell'età del bronzo. L'esempio delle terramare". Padusa 32-33: 47-67.

Quagliati, Q. 1903: "Ripostigli di bronzi arcaici del territorio di Taranto (Terra d'Otranto)". Bullettino di Paletnologia Italiana 29: 108-120.

Rockwell, P. 1989: Lavorare la pietra: Manuale per l'archeologo, lo storico dell'arte e il restauratore. La Nuova Italia Scientifica. Roma.

Rybczynski, W. 2001: One Good Turn: A Natural History of the Screwdriver and the Screw. Scribner. London.
Säflund, G. 1939: Le terremare delle provincie di Modena, Reggio Emilia, Parma, Piacenza. Almqvist \& Wiksells boktryckeri. Uppsala.

Stocks, D.A. 2003: Experiments in Egyptian Archaeology. Stoneworking technology in Ancient Egypt. Routledge. London.

Ugas, G. y Usai, L. 1987: "Nuovi scavi nel santuario nuragico di S. Anastasia di Sardara”. En La Sardegna nel Mediterraneo tra il secondo e il primo millennio a. C. Atti del Secondo Convegno di Studi "Un millennio di relazioni fra la Sardegna e i paesi del Mediterraneo" (Selargius-Cagliari 1986): 167-218. Cagliari.

Zancani Montuoro, P. 1977: "La leggenda di Epeo". Atti e Memorie della Società Magna Grecia n.s. 15-17: 93-106.

Zanini, A. (ed.) 1997: Dal Bronzo al Ferro. Il II millennio a.C. nella Toscana centro-occidentale. Comune di Livorno. Livorno. 